\title{
HIGHLIGHTS
}

$\mathrm{BPH}$

\section{Reduced rate of SUI after anteroposterior dissection HoLEP}

Holmium-laser enucleation of the prostate (HoLEP) is a widely used treatment for benign prostatic hyperplasia $(\mathrm{BPH})$, particularly in men with larger adenomas (those exceeding $100 \mathrm{~g}$ ). Numerous reports have indicated a relatively high rate (up to $44 \%$ ) of postoperative transient stress urinary incontinence (SUI) in men treated with this technique. Fumiyasu Endo and colleagues at St Luke's International Hospital, Tokyo, have adapted the standard HoLEP procedure (Gilling's method) with the aim of reducing the risk of postoperative SUI. They have dubbed their adaptation 'anteroposterior dissection HoLEP'.

The authors speculated that the antegrade dissection used in open prostatectomy - a procedure with a low risk of SUI (approximately 5\%) -is of major importance for preserving the structural integrity of the urethral sphincter. Their newly developed HoLEP technique differs from Gilling's method (which involves retrograde dissection of the prostate lobes) by employing an anteroposterior approach to dissection of the lateral lobes of the gland. They compared the outcomes of these two HoLEP procedures in 68 consecutive patients with $\mathrm{BPH}$ at a single center.

The first 31 patients underwent HoLEP according to Gilling's method between January and July 2008, and the remaining
37 patients underwent anterioposterior dissection HoLEP between July and December 2008. The two patient groups did not differ significantly at baseline in terms of age, estimated adenoma volume, international prostate symptom score (IPSS), quality of life score or uroflowmetry, including voided urine volume, peak flow rate and postvoid residual volume. All operations were performed by the same surgeon, who was experienced with HoLEP.

Mean operative time, resected prostate volume and decreases in hemoglobin level were similar in both groups. The patients returned to the hospital 2 weeks after surgery, and were evaluated for the presence of SUI. Patients were considered to have complete urinary control if they required no incontinence pads; SUI was defined as use of one or more pads. A significantly larger proportion of patients in the standard HoLEP group had SUI compared with the anteroposterior dissection HoLEP group (25.2\% versus $2.7 \% ; P=0.013)$. In addition, the latter group showed markedly better peak urine flow rates ( 19.8 versus $13.0 \mathrm{ml} / \mathrm{s} ; P=0.002$ ) and quality of life scores ( 1.5 versus 2.4 ; $P=0.02)$ compared to the standard HoLEP group.

The authors conclude that the introduction of their modified HoLEP procedure resulted in a significant decrease in the rate of postoperative

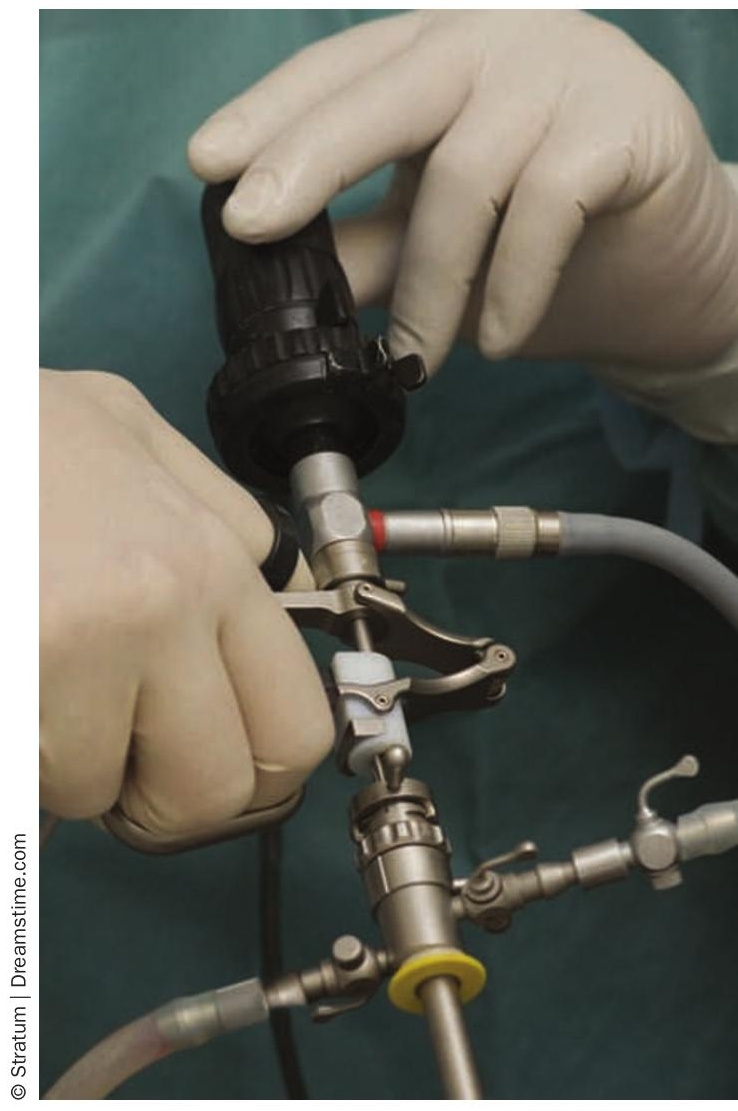

SUI at their center, with improved peak urine flow rates and quality of life scores. They also claim that their technique removes some of the complexity from the procedure, which might, they say, shorten the learning curve for surgeons.

\section{Nick Warde}

Original article Endo, F. et al. Anteroposterior dissection HoLEP: a modification to prevent transient stress urinary incontinence. Urology doi:10.1016/j.urology.2010.03.071 